\title{
Nuevo registro de Leptosalenia barredai Forner, 2014 (Echinoidea), procedente del Albiense de la cuenca Vasco-Cantábrica
}

\section{New occurrence of Leptosalenia barredai Forner, 2014 (Echinoidea) in the Lower Albian of the Basque-Cantabrian Basin (Western Pyrenees)}

Carmen Aranguren' ${ }^{1}$, Txema Moreno ${ }^{1}$, Enric Forner ${ }^{2 *}$

*

\section{Resumen}

Se da la segunda cita de Leptosalenia barredai Forner, 2014. El equínido, de la familia Saleniidae L. Agassiz 1838, se ha encontrado en el cabo de Ajo (Cantabria), procedente de una unidad de calizas y margocalizas marinas someras con corales del Albiense inferior de la cuenca VascoCantábrica. La especie sólo se conocía en su localidad tipo de Traiguera (cuenca del Maestrat), procedente de las capas de arenisca estuarinas del Albiense inferior y medio. Asimismo se establecen las diferencias entre L. barredai y L. faringdonensis Smith \& Wright, 1990. Paralelamente se ofrece también una nueva cita del coral Actinoseris sp., (Cnidaria) del Albiense de Traiguera descrito con nomenclatura abierta por Reig (1993).

Palabras clave: Echinoidea, Leptosalenia barredai, Albiense, cuenca Vasco-Cantábrica.

\begin{abstract}
A new occurrence of Leptosalenia barredai Forner, 2014 is presented. The echinoid is a component of the family Saleniidae 1838 L. Agassiz. It was found at Cape Ajo (Cantabria province, N Spain), in marly limestones of the Lower Albian age (Basque-Cantabrian Basin, Western Pyrenees). The species was only known in the type locality of Traiguera (Maestrat Basin, E Spain), from Lower-Middle Albian sandstone beds. The differences between L. barredai and L. faringdonensis Smith \& Wright, 1990 are established. Additionally we present a new occurrence of Actinoseris sp. (Cnidaria), a coral described by Reig (1993) in open nomenclature from the Albian of Traiguera.
\end{abstract}

Key words: Echinoidea, Leptosalenia barredai, Albian, Basque-Cantabrian Basin.

\footnotetext{
1 Ateneu de Natura

Elkano, 9-2 C 488992 Getxo

2 Ateneu de Natura

Sant Roc, 125 3r 5a • 12004 Castelló de la Plana

* Correspondencia: fornervalls@gmail.com
} 


\section{Laburpena}

Leptosalenia barredai Forner, 2014 espeziearen bigarren aipua argitaratzen da. Saleniidae L. Agassiz 1838 familiako ekinido hau Ajoko lurmuturrean aurkitu da (Kantabria), arro EuskalKantauriarreko Behe Albiarreko koraletako kareharri eta marga-kareharrizko itsas azaleko unitateetan. Espeziea soilik Traiguerako inguru tipoan ezagutzen zen (Maestrateko arroan), Behe eta Erdi Albiarreko paduretako hareharri geruzetan jatorria duena. Aldi berean L. barredai eta L. faringdonensis Smith \& Wright, 1990 espezieen arteko desberdintasunak finkatzen dira. Horrekin batera Traiguerako Albiarrean Actinoseris sp., (Knidario) koral espeziaren aipu berri bat deskribatzen da, Reig (1993) ren nomenklatura irekia erabiliz.

Gako hitzak: Echinoidea, Leptosalenia barredai, Albiense, arro Euskal-kantauriar.

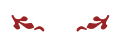

\section{Introducción}

Dentro de los trabajos de campo de un estudio que se está realizando sobre el género Goniopygus en la cuenca Vasco-Cantábrica (CVC) se han encontrado dos ejemplares de un equinoideo de la familia Saleniidae en los niveles albienses del cabo de Ajo (Bareyo, Cantabria), que se han clasificado como L. barredai, lo que supone la segunda cita mundial para la especie.

Leptosalenia barredai Forner, 2014, es un equínido descrito en la cuenca del Maestrat (Salas \& Guimerà, 1996, 1997) del que sólo se conocía su localidad tipo: Traiguera (el Baix Maestrat, Castelló). Tiene asignada una edad del Albiense inferior y medio y habitaba un ambiente de arrecifes coralinos, en un mar de aguas cálidas y someras (Forner, 2014 a).

Los estudios sobre el Albiense de la península Ibérica no son muy abundantes. En la cuenca del Maestrat se limitan exclusivamente a los yacimientos de Traiguera y Cabanes (Canerot \& Collignon, 1981; Reig \& Calzada, 1993; Forner, 2014 a, 2014 b).

Sobre la macro fauna albiense de la CVC, se dispone del trabajo sobre corales de la zona donde ha aparecido L. barredai de Baron-Szabo \& Fernández-Mendiola (1997). Asimismo, hay varias publicaciones, sobre ammonites (e.g., López-Horgue et al., 2009), decápodos (e. g., López-Horgue, 2009), braquiópodos (e. g., Viera \& Calzada, 1991) y moluscos (e. g., López-Horgue et al., 1998). Sin embargo no hay publicaciones específicas sobre equínidos del Albiense de la CVC.

El cabo de Ajo se encuentra en el centro de la cordillera Cantábrica en uno de los puntos más septentrionales de la península Ibérica, dentro del municipio de Bareyo en la comunidad de Cantabria. El yacimiento se encuentra en la parte noroeste de la CVC (Rat, 1959, 1988). Esta cuenca de rift estuvo activa desde el Triásico hasta el final del Eoceno, con dos etapas sucesivas principales de diferente intensidad (Rat, 1988). La segunda se caracteriza 
por una importante extensión continental durante el Aptiense y el Albiense, hecho que permitió el depósito de grandes espesores de sedimento, especialmente en el centro de la CVC (más de 7000 m; García-Mondéjar et al., 2004). En la zona de estudio se depositaron hasta 900 m de sedimentos durante el intervalo Aptiense-Albiense (e. g., Wilmsen, 1997). El nivel donde ha aparecido L. barredai es una caliza margosa con abundante fauna de corales, braquiópodos, gasterópodos y orbitolinas, situadas hacia el metro 173 de la columna estratigráfica levantada en ese zona por Baron-Szabo \& Fernandez-Mendiola (1997), cuya edad corresponde a un Albiense inferior.

\section{Sistemática}

En este apartado se ha seguido a Smith \& Wright (1990) y se ha consultado también a Kroh \& Smith (2010). En las abreviaturas usadas en la lista de sinonimias se han seguido las propuestas de Mattheus (1973): $v$ = ejemplar que ha sido estudiado por los autores. Las mediciones se han efectuado con un pie de rey digital y las medidas se dan redondeando a décimas de $\mathrm{mm}$. Las medidas tomadas han sido el diámetro (D), la altura (h), el diámetro del peristoma (dp) y el diámetro del disco apical (dda), que, al ser pentagonal, se ha medido desde el vértice de la placa ocular I hasta el centro de la arista opuesta. Todas las medidas se dan en $\mathrm{mm}$.

Se ha dispuesto de 2 ejemplares. El ejemplar figurado se deposita en el Museo Luberri (ML) en Oiartzun (Gipuzkoa) con el número de registro LUB-EG-479; el otro se conserva en la colección particular de Moreno \& Aranguren (CMA), en Getxo (Bizkaia).

Orden CALYCINA Gregory, 1900

Familia SALENIIDAE L. Agassiz, 1838

Subfamilia SALENIINAE L. Agassiz, 1838

Tribu SALENNINI L. Agassiz, 1838

Género Leptosalenia Smith \& Wright, 1990

Especie tipo: Salenia prestensis Desor, 1856 por designación original.

\section{Leptosalenia barredai Forner, 1914}

Figura $1 \mathrm{~A}-\mathrm{F}$

v 2014 Leptosalenia barredai Forner: 8, fig. 3 A-F, fig. 4 i fig. 5 A.

Holotipo conservado en el Museu de Geologia - Museu de Ciències Naturals de Barcelona (MGB) con el número de registro MGB 59964 (fig. 1 E-F).

Diagnosis: Leptosalenia pequeña con el disco apical cónico de contorno pentagonal de bordes casi rectos. Placas oculares de contorno romboidal.

Para la descripción se remite al artículo original (Forner, 2014). 


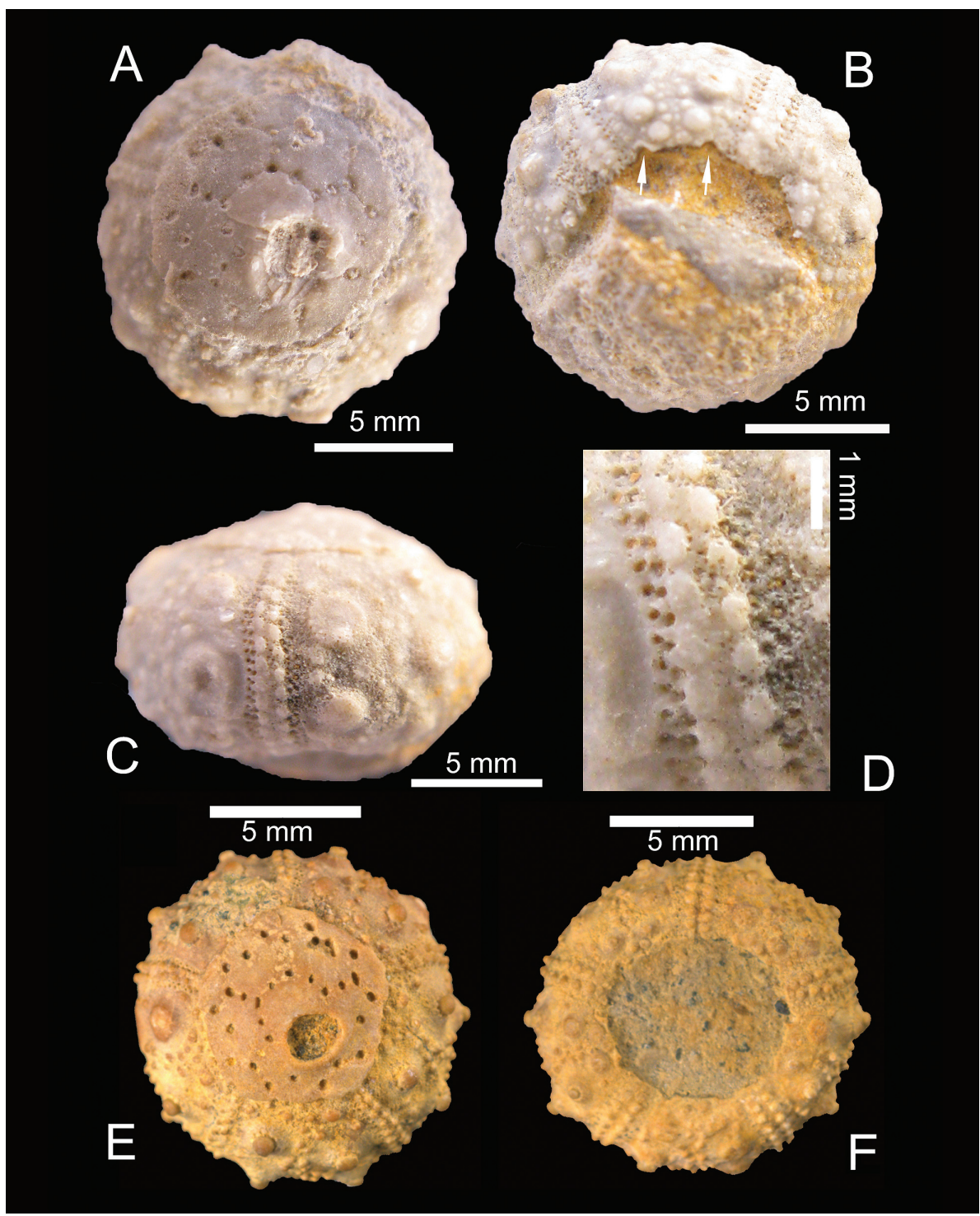

Fig.1.- Leptosalenia barredai, Forner, 2014, ejemplar LUB-EG-479, Museo Luberri, del Albiense inferior de Ajo (cuenca Vasco-Cantábrica), A: vista apical; B: vista oral; C: vista lateral; D: detalle tubérculos ambulacrales. Holotipo, MGB-59964, Museu Geològic de Barcelona, del Albiense inferior-medio de Traiguera (cuenca del Maestrat); E: vista apical; F: vista oral. Las flechas indican las escotaduras branquiales. Fotos: E. Forner. Montaje: V. Gual.

Fig. 1.- Leptosalenia barredai, Forner, 2014, LUB-EG-479, Museo de Luberri, from Albian of Ajo (BasqueCantabrian Basin, N Spain); A: apical view; B: oral view (The arrows indicate buccal slits); C: lateral view; D: detail of ambulacral tuberculation. Holotype MGB-59964, Museu Geològic de Barcelona, from Albian of Traiguera (Maestrat Basin, NE Spain). E: apical view; F: oral view. Pictures: E. Forner. Assembly: V. Gual. 
Medidas. El ejemplar figurado, LUB EG-479, mide D: 11,7; h: 8,3 mm; h/D: 71\%; diámetro del disco apical: 7,5; dda/D: 64\%; diámetro del peristoma: 6,9; dp/D: 59\%. El ejemplar de la CMA mide D: 13,6; h: 9.5 mm; h/D: 68,6\%, es el más grande registrado (tabla 1).

\begin{tabular}{|ccccccc|}
\hline & LUB & CMA & \multicolumn{4}{c|}{ ESTUDIO FORNER 2014 } \\
& EG-479 & CMA-1424 & M & Max & Min & $n$ \\
\hline D (mm) & 11,7 & 13,6 & 10,4 & 13,1 & 7,8 & 21 \\
$h(\mathrm{~mm})$ & 8,3 & 9,4 & 7,3 & 9,3 & 4,7 & 19 \\
h/D (\%) & 70,9 & 67,9 & 68,8 & 84,1 & 56,5 & 18 \\
Dpt (mm) & 6,9 & 6,7 & 4,9 & 6,0 & 3,8 & 10 \\
Dpt/D (\%) & 59,0 & 49,3 & 48,8 & 56,1 & 40,4 & 9 \\
\hline
\end{tabular}

Tabla 1.- Medidas de los ejemplares de Leptosalenia barredai Forner, 2014 del Albiense del cabo de Ajo (Bareyo, cuenca Vasco-Cantábrica) y comparación con los ejemplares de la localidad tipo (Traiguera, cuenca del Maestrat).

Table 1.- Dimensions of Leptosalenia barredai Forner, 2014 from Albian of the Ajo Cab (Bareyo, Basque-Cantabrian Basin, N Spain) and compared with specimens from the type locality (Traiguera, Maestrat basin, N Spain).

Se han podido clasificar los ejemplares de Ajo como L. barredai porque las escotaduras branquiales poco profundas y en forma de " $U$ " (fig. 1 B y E) separan el material estudiado de L. prestensis (Desor, 1856) y L. faringdonensis Smith \& Wright, 1990, que las tienen profundas y en forma de V (Smith \& Wright, 1990: PI. 37, fig. 1C). L. barredai no presenta entrantes en el perímetro del disco apical entre las placas genitales y las oculares mientras que sí los presentan las otras dos especies, más acusados en prestensis que en faringdonensis.

L. faringdonensis también se diferencia de L. barredai por la placa madrepórica, en la cual la depresión con los hidroporos es pequeña (como dos veces el tamaño de un poro genital frente a más de cinco en barredai) y entra en contacto con la placa ocular III; el poro genital de la placa 2 es externo a la depresión madrepórica mientras que siempre son internos, cuando existen, en barredai; las fosetas entre las suturas de las placas de L. faringdonensis son poco desarrolladas o inexistentes en los contactos entre tres placas; la relación altura diámetro es inferior, tiene más tubérculos primarios interambulacarles y menos ambulacrales y el disco apical, en perfil, no desarrolla un cono prominente como en L. barredai. Una síntesis de las diferencias se recoge en la tabla 2.

\section{Otras consideraciones paleontológicas}

Este hallazgo es concordante con la existencia de conexión entre las cuencas Vasco-Cantábrica y del Maestrat durante el Albiense (fig. 2).

El ambiente que se puede deducir para este nuevo hallazgo de L. barredai sería de plataforma carbonatada, salinidad plenamente marina (la propia presencia de equínidos, 


\begin{tabular}{|lccc|}
\hline Diferencias & barredai & faringdonensis & prestensis \\
\hline Diámetro máximo registrado & 13,6 & 12,5 & 20,0 \\
Relación altura/diámetro (H/D) & $69 \%$ & $59 \%$ & $61 \%$ \\
Angulo disco apical vista lateral & $120^{\circ}$ & $150^{\circ}$ & $170^{\circ}$ \\
La zona deprimida con hidroporos llega a la placa ocular III & No & Sí & Sí \\
Tamaño depresión hidropors respecto gonoporus & $>6$ & 2 & $>4$ \\
Posición poros genitales placa 2, dentro o fuera depresión & dentro & fuera & fuera \\
Fosetas contactos 3 placas regulares y bien desarrolladas & Sí & No & Sí \\
Forma de las escotaduras branquiales & U & V & V \\
Profundidad escotaduras branquiales & poca & mucha & mucha \\
Número placas interambulacrales (PI) & $3 / 4$ & 5 & $5 / 6$ \\
Tubérculos principales ambulacros & 19 & $14 / 15$ & 16 \\
Perímetro disco apical & pentagonal & subpentagonal & circular \\
Perímetro p. oculares & romboidal & triangular & subtriangular \\
Borde externo p. oculares & rectos & convexo & concavo \\
Posición del ámbito respecto altura desde cara oral & $1 / 3$ & $1 / 2$ & $1 / 2$ \\
El ancho de los ambulacros se expande cerca peristoma & No & Sí & No \\
Hendidura contacto p. ocular y p. genital & No & Sí & Sí \\
Tamaño de les fosetas suturales & No & variable & variable \\
Borde externo p. oculares excede a genitales & Sí \\
\hline
\end{tabular}

Tabla 2.- Diferencias principales entre Leptosalenia barredai Forner, 2014 del Albiense de España, L. faringdonensis Smith \& Wright, 1990, del Albiense de Gran Bretaña (Datos de Smith \& Wright, 1990) y L. prestensis (Desor, 1856) del Aptiense de Francia y España (datos de Smith \& Wright, 1990).

Table 2.- Main differences between Leptosalenia barredai Forner, 2014 from Albian of Spain, L. faringdonensis Smith \& Wright, 1990, from Albian of Britain (according to Smith \& Wright, 1990) and L. prestensis (Desor, 1856) from the Aptian of France and Spain (according to Smith \& Wright, 1990).

corales y de braquiopodos lo indica), de poca profundidad y aguas cálidas (dominancia de corales).

La asociación de fósiles tanto en número de ejemplares como en diversidad de especies está dominada por el filum Cnidaria (Baron-Zsabo \& Fernández-Mendiola, 1997). Estos autores consideran que el yacimiento de Ajo representa un ambiente subtropical de aguas someras y con alta energía. En conclusión, un ambiente muy similar al que se indica para su localidad tipo (Forner, 2014 a) en la cuenca del Maestrat, aunque con menor energía 


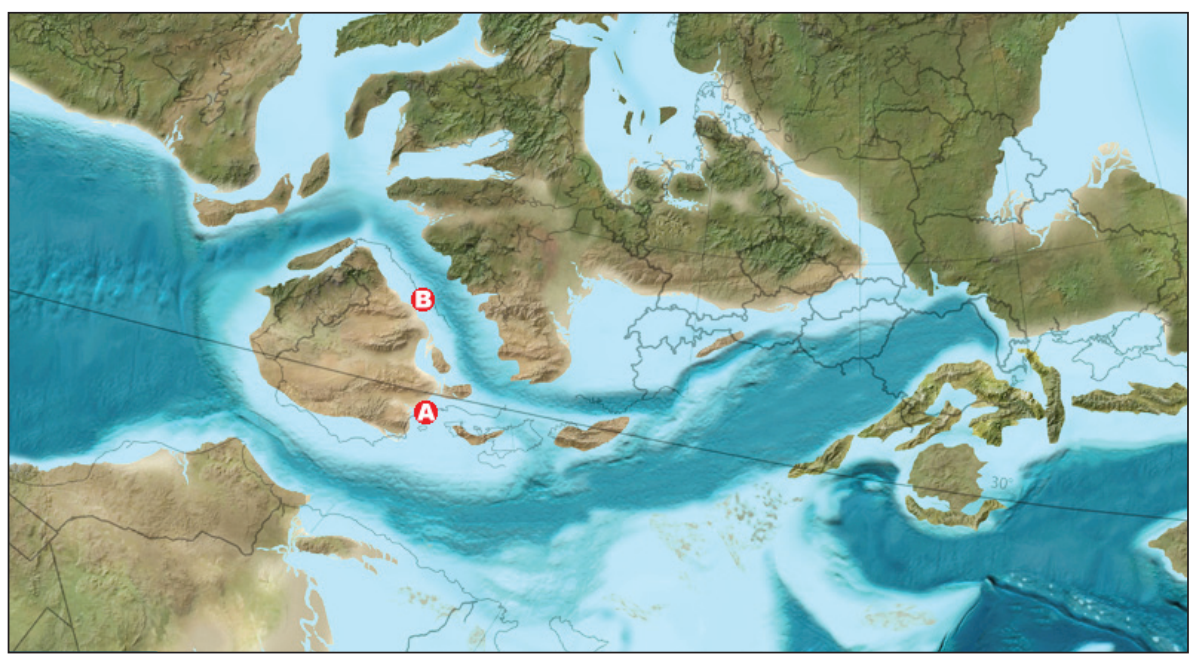

Fig. 2.- Paleo mapa de la parte central del Tethys al final del Albiense. Los puntos indican las cuencas donde ha aparecido Leptosalenia barredai Forner, 2014. A: cuenca del Maestrat; B: cuenca Vasco-Cantábrica; Marrón: tierras emergidas, Blanco: mar, plataforma continental; Azul: mar profundo. Fuente: Blakey, NAU Geology, 2014, modificado.

Fig. 2.- Palaeomap showing the central part of the Tethys towards the end of Albiense The lettered dots indicate the marine basins where Leptosalenia barredai Forner, 2014 was foound. A: Maestrat Basin; B: Basque-Cantabrian Basin; Brown: landmasses; White: continental shelf; Blue: deep sea. Source: Blakey, NAU Geology, 2014, as amended.

en este último. Entre los gasterópodos presentes en Ajo, que están en fase de estudio, se ha determinado Helicacanthus octavius (d'Orbigny, 1850), que también aparece en la fauna asociada a L. barredai en su localidad tipo. Tambien han aparecido en Ajo como fauna acompañante de L. barredai 2 ejemplares del coral solitario de pequeñas dimensiones Actinoseris sp. descrito por Reig (Reig \& Calzada, 1993) en nomenclatura abierta, que no se cita en el trabajo de Baron-Zsabo \& Fernández-Mendiola (1997); dicho coral acompañaba también a L. barredai en el yacimiento de Traiguera.

La edad que se asigna a la localidad nueva del cabo de Ajo para L. barredai es del Albiense Inferior según Baron-Zsabo \& Fernández-Mendiola (1997).

De los dos ejemplares estudiados de Ajo, uno excede los máximos de diámetro y altura de los de Traiguera (n: 21). Son muestras pequeñas pero es posible que exista una diferencia de tamaño entre los ejemplares de las dos localidades, que se puede asimilar dentro de las diferencias intraespecíficas.

\section{Agradecimientos}

A Mikel López-Horgue, por sus indicaciones sobre la geología de la CVC. A Vicent Gual por la composición de las figuras. A Carl Nugent por las correcciones de los textos en inglés. 


\section{Bibliografía}

- Baron-Szabo, R. C., Fernández-Mendiola, P. A. 1997. Cretaceous scleractinian corals from the Albian of Cabo de Ajo (Cantabria Province, N-Spain). Paläontologische Zeitschrift 71 (1/2): 35-50.

- Blakey, R. Colorado Plateau Geosystems, Inc. Paleography Library- Regional Paleography- European Paleography- Paleographic and Tectonic History of Europe- European Paleographics Maps - Mesozoic-Early Cretaceous (125 Ma). Disponible en: http://www.cpgeosystems.com/ (Consulta: 21-09-2014).

- Canerot, J., Collignon, M. 1981. Le faune albienne de Traiguera (province de Castellon, Espagne). Documents du Laboratoire de Géologie de I'Université de Lyon, Hors Série 6: 227-249.

- Forner, E. 2014a. Una nova espècie del gènere Leptosalenia Smith \&Wright, 1990 (Echinoidea: Saleniidae) de l'Albià de Traiguera (Conca del Maestrat, NE de la península Ibèrica). Treballs del Museu Geol. Barcelona 20: 5-13.

- Forner, E. 2014b. Calzadina segurai gen. nov., sp. nov. (Mollusca: Gastropoda) de l'Albià de Cabanes de l'Arc (la Plana Alta, conca del Maestrat). Nemus 4: 17-26.

- García-Mondéjar, J., Fernández-Mendiola, P. A., Agirrezabala, L. M., Aranburu, A., López-Horgue, M. A., Iriarte, E. and Martinez de Rituerto, S. 2004. El Aptiense-Albiense de la Cuenca Vasco-Cantábrica. En: Geología de España. J.A.Vera (Ed.): 291-296. SGE-IGME. Madrid.

- Kroh, A, Smith, A.B. 2010. The filogeny and classification of post-Paleozoic echinoids. J. Syst. Palaeontol. 8 (2): 147-122.

- López-Horgue, M.. A. 2009. New ocurrences of Meyeria magna M?Coy, 1849 (Decapoda, Mecochiridae) in the early Aptian and early Albian of the Basque Cantabrian Basin (North Spain). Geogaceta 47: 25-28.

- López-Horgue, M. A., Aranburu, A., Gómez-Pérez, I., Fernández-Mendiola, P.A., GarcíaMondéjar, J., Calzada, S. 1998. Fauna bentónica de ambientes siliciclásticos de mar somero. Albiense superior de Bizkaia y Araba (Península Ibérica). Batalleria 8: 37-52.

- López-Horgue, M. A., Owen, H. G., Aranburu, A., Fernández-Mendiola, P.A., García-Mondéjar, J. 2009. Early late Albian (Cretaceous) of the central region of the Basque-Cantabrian Basin, northern Spain: biostratigraphy based on ammonites and orbitolinids. Cretaceous Res. 30: 385-400.

- Matthews, S.C. 1973. Notes on open nomenclature and synonymy lists. Paleontology 16(4): 713-719.

- Rat, P. 1959. Les Pays crétacés basco-cantabriques. Publ. Univ. Dijon. Dijon.

- Rat, P. 1988. The Basque-Cantabrian Basin Between the Iberian and European plates som facts but still many problems. Rev. Soc. Geol. España 1 (3-4): 327-348.

- Reig, J. M., Calzada, S. 1993. Nuevos datos sobre la fauna albiense de Traiguera (Castellón). Cuad. Geol. Ibérica 17: 371-391. 
- Salas, R., Guimerà, J. 1996. Rasgos estructurales principales de la cuenca cretácica inferior del Maestrazgo (Cordillera Ibérica oriental). Geogaceta 20(7): 1704-1706.

- Salas, R., Guimerà, J. 1997. Estructura y estratigrafía secuencial de la cuenca del Maestrazgo durante la etapa de Rrift Jurásica superior-Cretácica inferior (Cordillera Ibérica Oriental). Bol. Geol. Min. 108(4-5): 393-402.

- Smith, A. B., Wright, C. W. 1990. British Cretaceous Echinoids. Part 2. Echinothurioida and Stirodonta (1, Calycina). Monograph of the Palaeontolographical Society 583 (143): 101-198.

- Viera, L.I., Calzada, S. 1991. Un yacimiento de braquiopodos en el Albiense Inferior (complejo urgoniano) del macizo de Aitzgorri (Guipuzcoa). Munibe, Cienc. nat. 43: 9-19.

-Wilmsen, M. 1997. Das Oberalb und Cenoman im Nordkantabrischen Becken (Provinz Kantabrien, Nordspanien). (Berliner Geowissenschaftliche Abhandlungen, reihe E; 23). 